\title{
Limitaciones del modelo constructivista en la enseñanza-aprendizaje de la Unidad Educativa Salcedo, Ecuador
}

\author{
Amores Torres, José Luis; Ramos Serpa, Gerardo \\ Limitaciones del modelo constructivista en la enseñanza-aprendizaje de la Unidad Educativa Salcedo, Ecuador \\ Revista Educación, vol. 45, núm. 1, 2021 \\ Universidad de Costa Rica, Costa Rica \\ Disponible en: http://www.redalyc.org/articulo.oa?id=44064134006 \\ DOI: https://doi.org/10.15517/revedu.v45i1.41009
}

Esta obra está bajo una Licencia Creative Commons Atribución-NoComercial-SinDerivar 3.0 Internacional. 


\title{
Limitaciones del modelo constructivista en la enseñanza-aprendizaje de la Unidad Educativa Salcedo, Ecuador
}

\author{
José Luis Amores Torres \\ Unidad Educativa Salcedo, Ecuador \\ joselo13amores@gmail.com \\ (iD http://orcid.org/0000-0001-5053-7154 \\ Gerardo Ramos Serpa \\ Universidad Regional Autónoma de los Andes, Ecuador \\ gramosserpa@gmail.com \\ (iD http://orcid.org/0000-0003-3172-555X
}

Limitations of a Constructivist Teaching-Learning Model At the Salcedo, Ecuador Educational Unit

\author{
DOI: https://doi.org/10.15517/revedu.v45i1.41009 \\ Redalyc: http://www.redalyc.org/articulo.oa?id=44064134006
}

Recepción: 14 Abril 2020

Aprobación: 21 Agosto 2020

\section{Resumen:}

La presente investigación se desarrolló en la Unidad Educativa Salcedo, cantón el cual lleva su mismo nombre, ubicado en la provincia de Cotopaxi, Ecuador. Este trabajo presenta resultados de un análisis basado en la pedagogía constructivista del proceso de enseñanza-aprendizaje, cuyo propósito fue tener una visión clara sobre el tipo de metodología utilizada dentro del aula de clases por parte de las maestras y los maestros. Se pudo verificar que este interviene de manera significativa al ejecutar algunos cambios, de la misma forma que elementos y técnicas son los más apropiados para lograr un mejor conocimiento en el alumnado. Se contó con la participación de docentes de noveno año de Educación General Básica, del período académico 2019 - 2020, en las asignaturas esenciales del tronco común en el currículo educativo ecuatoriano (Matemáticas, Lengua y Literatura, Ciencias Naturales y Estudios Sociales), donde se pudo detectar distintos problemas de aprendizaje como distracción, aburrimiento, falta de interés, entre otros, lo cual dificultaba su aprendizaje; posteriormente, se efectuaron medidas correctivas. Para este análisis se utilizó un enfoque de estudio cualitativo con un diseño de investigación-acción. Además, se elaboraron entrevistas al cuerpo docente mediante un formulario online de Google, las cuales permitieron diagnosticar el conocimiento a nivel teórico de la pedagogía constructivista, lo que a su vez se corroboró con una ficha de observación donde se pudo constatar cómo son las clases de cada docente sin previo aviso. Los resultados más relevantes indican que el profesorado, en teoría, conoce los conceptos de la pedagogía constructivista, sin embargo, al momento de trasladarlos al aula, ello no se evidencia, por lo que se recomienda a las autoridades capacitación continua, el dialogo con el personal docente para que utilicen el modelo constructivista, que usen nuevas formas de enseñanza dentro del aula de clases y rompan el temor que tienen para aplicarlo.

Palabras claVe: Modelo pedagógico, Enseñanza-aprendizaje, Pedagogía Constructivista, Educación General Básica.

\section{Abstract:}

This study was conducted at the Salcedo Educational Unit, in the province of Cotopaxi, Ecuador and is based on a constructive pedagogy analysis of teaching and learning. The objective of the study was to observe the different types of methodologies teachers use in classrooms and, as we corroborated, their impact on change and the elements and techniques which are essential to helping students achieve better knowledge acquisition. Participants included Ninth grade Basic General Education Teachers for the 2019-2020 academic year, responsible for teaching the essential core subjects of the Ecuadorian Educational Curriculum (Mathematics, Language and Literature, Natural Sciences and Social Studies). If learning weaknesses were detected, such as distraction, boredom or lack of interest, which hindered learning, corrective measures were then taken. The study was based on a qualitative approach with an action-research design. Teachers were interviewed using an online Google form, allowing us to gauge their theoretical knowledge of constructivist pedagogy. Observation of the teachers while in class was then carried out to corroborate their knowledge and performance. It was noted, that many of these results noted in the theoretical evaluation were not applied in the classroom, which emphasizes the need for further training and open dialogues with the teachers. It is recommended that school authorities provide continuous training and discussions with the teaching staff so that they may be able to use the constructivist mode in class, learn new teaching techniques and be less fearful of applying them in the classroom.

KEYwords: Pedagogical Model, Teaching - Learning, Constructivist Pedagogy, Basic General Education. 


\section{INTRODUCCIÓN}

La Unidad Educativa Salcedo es una entidad de educación pública que ofrece bachillerato general unificado y bachillerato internacional, ubicada en el cantón Salcedo, Ecuador, y con 68 años de experiencia educativa. La formación que brinda esta institución es la Educación General Básica (E.G.B), Bachillerato General Unificado (B.G.U) y Bachillerato Internacional (BI), pero el presente estudio está basado en los novenos años del período lectivo 2019-2020, debido a la problemática de la carencia de un modelo educativo adecuado en el proceso de enseñanza-aprendizaje, principalmente en las materias básicas que se imparten, como son: Ciencias Naturales, Estudios Sociales, Lengua y Literatura, y Matemáticas.

El presente trabajo se ha preparado de forma pertinente, con el propósito de mejorar y poner a disposición de los docentes un instrumento basado en el conocimiento que debe existir sobre la aplicación del modelo constructivista para la enseñanza-aprendizaje, para que este pueda ser puesto en práctica de manera adecuada y eficaz, pues la educación requiere de personas dispuestas a asumir retos con el afán de mejorar la calidad de la educación.

Esta investigación, asimismo, tiene gran importancia a nivel institucional porque se va a analizar cómo el modelo pedagógico constructivista interviene de manera favorable dentro del proceso de enseñanzaaprendizaje en el estudiantado de noveno grado de la Unidad Educativa Salcedo, esto con el fin de buscar soluciones a las posibles deficiencias académicas que pueden presentarse en el alumnado, teniendo en cuenta los lineamientos institucionales y de acuerdo con las necesidades de la comunidad educativa.

El sujeto principal en la educación es el estudiante, de modo que este trabajo lo beneficiará porque el profesorado podrá poner en práctica técnicas y métodos del modelo constructivista para mejorar la enseñanza-aprendizaje, lo cual permitirá que cada estudiante sienta mayor interés en las clases de cada asignatura y mejore su rendimiento académico.

Para el investigador tiene gran relevancia porque obtendrá nuevos conocimientos sobre el modelo constructivista, y de esta manera podrá aplicarlos en clase y ser un guía para sus compañeros. Para la sociedad y comunidad educativa tiene gran importancia debido a que la investigación va a ayudar a transmitir nuevos conocimientos para mejorar la calidad educativa con la búsqueda de nuevas formas de enseñanza-aprendizaje donde todos sean beneficiados y así tener jóvenes preparados para que sirvan a la sociedad.

El estudio abarca el contexto teórico práctico, tomando en cuenta como teoría el modelo pedagógico constructivista, por lo que el objetivo principal del presente estudio consiste en analizar la aplicación del modelo pedagógico constructivista en el proceso de enseñanza-aprendizaje de la Unidad Educativa Salcedo, Ecuador, con la finalidad de establecer las limitaciones en su aplicación.

De la misma manera, entre sus objetivos específicos se encuentran:

- Describir de qué manera influye el modelo pedagógico constructivista en el proceso de enseñanza aprendizaje.

- Reconocer los distintos procesos de enseñanza aprendizaje apropiados para estudiantes de noveno año de EGB de la unidad educativa Salcedo, Ecuador.

- Identificar qué dificultades de aprendizaje presenta el estudiantado del noveno año de E.G.B de la Unidad Educativa Salcedo, Ecuador.

Se ha sustentado como hipótesis el reconocimiento de que la aplicación del proceso de enseñanzaaprendizaje en las materias básicas del noveno grado son impartidas sin la necesidad de un modelo de aprendizaje establecido y la finalidad de esta es relacionar y utilizar el modelo constructivista por parte del cuerpo docente para perfeccionar la enseñanza-aprendizaje del alumnado. 


\section{Referente TEÓRICo}

\subsection{Antecedentes}

Otras investigaciones encontradas fuera del país abordan el estudio de la pedagogía constructivista, así como el trabajo de Vera (2009) elaborado en Medellín, este propone estrategias didácticas constructivistas, en las cuales el/la maestro/a sea el/la guía, quien propone actividades para el estudiantado que contribuyan a mejorar la participación y la responsabilidad de estos, así como asumir su compromiso con el aprendizaje debido a su bajo nivel académico y despreocupación.

Esto también lo desarrolla Domínguez (2010) en Boca del Rio Veracruz, este estudio se refiere al aprendizaje basado en problemas (ABP), el cual hace referencia a las estrategias constructivistas en la enseñanza de la geografía, en el cual indica que esta "favorece la secuencia y el ordenamiento en la forma de adquirir el aprendizaje, así como la autonomía para la búsqueda, selección, integración, reflexión y autoevaluación de la información" (p. 17).

A nivel nacional hay estudios similares donde se intenta ofrecer algo nuevo como el caso de Rosero (2017), ejecutado en la provincia de Cotopaxi, el cual hace referencia a la educación intercultural bilingüe y la forma de incluir el constructivismo para mejorar el aprendizaje del estudiantado, al mismo tiempo introducir para esto materiales que se encuentren en la zona, con el fin de romper esquemas memorísticos

De la misma forma, Serna (2011) relaciona métodos y técnicas de enseñanza que se pueden aplicar mediante el constructivismo para desarrollar el pensamiento lógico del alumnado, de tal forma que este sea el actor principal y el personal docente sea capaz de cambiar la manera de impartir su clase al trabajar con actividades que impliquen un aprendizaje significativo.

Robalino (2016) desarrolla su investigación en una unidad educativa de la ciudad de Ambato basado en el modelo pedagógico constructivista y cómo influye en el proceso de aprendizaje del estudiantado; al mismo tiempo, llega a la conclusión de que el cuerpo docente no lleva este proceso de aprendizaje en el aula, por lo que su clase se torna aburrida por la ausencia de otros elementos como material didáctico.

$\mathrm{Al}$ modelo constructivista también se adhiere el área de computación como el caso de Pérez y Morales (2012) quienes investigan cómo esta metodología puede usarse para su área de enseñanza para aplicar las técnicas y estrategias adecuadas, además de ofrecer al alumnado todos los recursos didácticos necesarios como imágenes, sonido, interactividad de los materiales multimedia para que el estudiantado sea el protagonista y dejar atrás la enseñanza conductista.

Todos estos trabajos son importantes porque proporcionan información y formas innovadoras de enseñanza que se pueden tener en cuenta como apoyo a investigaciones más contextuales.

\subsection{Modelos pedagógicos}

Es indispensable que la educación actual incorpore modelos acordes al contexto social que vive cada una de las Unidades Educativas; para ello se necesita el mejoramiento de estrategias, técnicas y preparación docente para llegar al pensamiento metacognitivo de las y los estudiantes. La enseñanza tradicional no ha podido resolver esta situación debido a los acelerados cambios tecnológicos, pues las nuevas políticas educativas en el país se encuentran muchas veces diseñadas y declaradas en papeles, pero se han incorporado lentamente en los espacios áulicos, y aún más lentos, en la capacitación docente (Kuiper, Murdock, Grant, 2010).

Actualmente, la educación está pasando por una serie de cambios en Ecuador, lo cual debe ser tomado en cuenta por los educadores para así reconocer que la educación tradicional terminó, que se usan nuevas formas de enseñanza-aprendizaje donde intervienen técnicas, métodos nuevos e incluso el uso de instrumentos electrónicos para poder llegar al estudiantado y que este pueda tener un aprendizaje significativo. 


\section{Durán (2015) menciona que}

enseñar estrategias no consiste en enseñar recetas en forma unívoca y algorítmica, sino debe hacer hincapié en los momentos críticos de la resolución, el educando debe ser consciente del por qué y en qué momento hacer uso de una estrategia u otra (p. 17).

Es necesario recalcar que la persona educadora no debe ir con una estrategia a impartir clases, debe tener más amplitud y flexibilidad, por ende, disponer de recursos que pueda utilizar en caso de ser necesario, porque muchas de las veces es preciso aplicar habilidades o destrezas diferentes o no previstas para que el estudiantado preste la debida atención e incluso se sienta a gusto en la clase.

\subsection{Modelo constructivista}

Dentro de este modelo básicamente se trata de ir construyendo el conocimiento de cada estudiante partiendo de elementos básicos, realidades y experiencias para así conseguir resaltar las habilidades y destrezas de cada uno de ellos, teniendo en cuenta el análisis de cada situación y su mejor forma de resolución.

Zapata-Ros, (2015) explican que

el estudiante no se limita a copiar el conocimiento, sino que lo construye (constructivismo) a partir de elementos personales, experiencia e ideas previas e implícitas, para atribuir significado (eso es también comprender) y representarse el nuevo conocimiento con sentido adquirido (el contenido del aprendizaje) (p. 75).

\subsection{Tipos de constructivismo}

\subsubsection{Constructivismo radical}

Establecer este tipo de constructivismo para muchos puede ser de gran beneficio, porque permite reconocer que se crean conocimientos, habilidades, valores, etc. de acuerdo con sus realidades vividas. Serrano y Pons (2011) afirman:

El constructivismo radical, cuyo máximo representante es Von Glasersfeld (1995), hace referencia a un enfoque no convencional del problema del conocimiento y del hecho de conocer y se basa en la presunción de que el conocimiento, sin importar cómo se defina, está en la mente de las personas y el sujeto cognoscente no tiene otra alternativa que construir lo que conoce sobre la base de su propia experiencia (p. 6).

Este tipo de constructivismo define que se encuentra principalmente en la mente de las personas y se crea sobre lo que conoce, este aspecto es importante pues forma parte de construir el conocimiento desde aspectos que pueden recordar y al mismo tiempo que recién descubren.

\subsubsection{Constructivismo cognitivo}

El constructivismo cognitivo, que parte esencialmente de la teoría piagetiana y que postula que el proceso de construcción del conocimiento es individual, desarrolla los análisis sobre estos procesos bajo tres perspectivas: la que conduce al análisis macrogenético de los procesos de construcción; la que intenta describir y analizar las microgénesis, y la vertiente integradora de estas dos posiciones (Serrano y Pons, 2011).

Este tipo de constructivismo está relacionado con todo lo que se refiere al pensamiento, el lenguaje que se aplique, el razonamiento del porqué de las cosas, la atención y la forma como se pueden resolver los problemas, entre otros; por lo tanto, es importante para que el estudiantado pueda desarrollar su intelecto, los conocimientos y, sobre todo, lo que existe a su alrededor. 
Saldarriaga, Bravo y Loor (2016) en su artículo hacen referencia a la teoría constructivista e indican lo siguiente:

La teoría constructivista de Jean Piaget no constituye para nada una solución simplista a un problema tan complejo como el desarrollo cognoscitivo, si se tiene en cuenta que el conocimiento se produce como un proceso complejo de construcción por parte sujeto en interacción con la realidad, no se trata del mero hecho de obtener respuestas, sino que lo verdaderamente importante es cómo se produce el aprendizaje (p. 130).

La teoría constructivista de Piaget tiene gran importancia y es la base con la cual se trabaja en una parte significativa de los centros educativos en la actualidad, la cual busca el desarrollo cognitivo del estudiantado y la construcción del saber a través de hechos reales, para que así exista el aprendizaje y desarrollen conocimientos reales.

Morales e Irigoyen (2016), refiriéndose al trabajo de Piaget, indican:

A su trabajo también se le ha denominado epistemología genética, ya que su interés investigativo fue la evolución mental en el niño, para determinar cómo se construye el conocimiento humano, en función de los procesos de naturaleza fisiológica y psicológica involucrados en el tránsito de un estadio de menor conocimiento a otro de mayor conocimiento. Por esta razón, la problemática a la que se aboca el constructivismo es la construcción del conocimiento (p. 28).

Es importante tener en cuenta aspectos genéticos para desarrollar los conocimientos e ir construyéndolos estos gradualmente, obteniendo así un proceso donde vayan aprendiendo desde lo más sencillo hasta lo más complejo, y de esa forma buscar un aprendizaje significativo.

\subsection{Enseñanza-aprendizaje}

Espejo y Sarmiento (2017) afirman: "las metodologías activas de enseñanza-aprendizaje tienen como fin último maximizar las probabilidades de que los estudiantes aprendan en una cierta situación implementada por el profesor" (p. 10). Sin embargo, esto se puede lograr de mejor manera cuando se incluye al estudiantado en situaciones de la vida real, donde se puedan ver involucrados y comiencen a buscar soluciones rápidas y situadas; además, es necesario que el/la docente tenga claro las metodologías que aplica en clase, porque son una base que el alumnado adopta para buscar una salida al problema.

Cuando se refiere a la enseñanza-aprendizaje, el profesorado debe tener en cuenta cómo se va a generar el conocimiento. Campos y Moya (2011) indican que

el proceso de enseñanza aprendizaje tiene como propósito esencial favorecer la formación integral de la personalidad del educando, constituyendo una vía principal para la obtención de conocimientos, patrones de conducta, valores, procedimientos y estrategias de aprendizaje (p. 2).

El cuerpo docente al momento de impartir clases debe ser consciente del propósito de la clase que va a desarrollar y del perfil de salida o resultados por alcanzar al culminar esta, donde enmarque la enseñanza; además, tener un estilo propio que esté relacionado al proceso de aprendizaje, para de esta forma obtener y promover el interés del estudiantado por aprender.

Para la UNESCO (2015),

esta es una realidad que los docentes requieren apoyarse en estrategias, que deben buscar la manera de crear planes y programas de estudios con evaluaciones que promuevan reducir las desigualdades en el aprovechamiento escolar para brindar a todos los niños y jóvenes la oportunidad de conseguir capacidades transferibles fundamentales (p. 11)

Es importante tener en cuenta que la realidad de cada institución es diferente, por lo que no debe apoyarse en ciertos parámetros estandarizados, sino también se debe guiar a las comunidades educativas de forma determinada y contextualizada, donde exista una buena comunicación entre padres de familia, estudiantes y docentes, para así poder resolver de manera oportuna problemas existentes dentro del ámbito educativo. 


\title{
2.6. Proceso de enseñanza aprendizaje por competencia
}

\author{
Andrade (citado en Marrero, 2017):
}

\begin{abstract}
Piensa que el proceso de enseñanza-aprendizaje por competencias implica la ruptura con prácticas, formas de ser, pensar y sentir desde una racionalidad en la que se concibe que la función de la universidad es enseñar, para producir formas de vida, cultura e ideología de estudios cargados de contenidos, que concibe la teoría desvinculada de la práctica y el profesor ocupa el rol hegemónico y absoluto en la dirección de este (p. 31).
\end{abstract}

De acuerdo con lo mencionado, el proceso de enseñanza-aprendizaje por competencias tendría un enfoque más personal hacia cada estudiante, debido a que se establecería un/a tutor/a, el cual tendría a su cargo la misión de enseñar planteando nuevas formas de aprendizaje bajo nuevos instrumentos, donde el estudiantado destaque su papel activo mediante su capacidad de demostrar la validez y aplicación de lo que aprende.

\section{Metodología}

\subsection{Enfoque}

El objetivo principal de esta investigación es analizar el proceso de enseñanza-aprendizaje y si este mantiene relación con el modelo pedagógico establecido en los documentos institucionales en la unidad educativa señalada, para ello el tipo de enfoque por utilizar es cualitativo, basado en tomar la información en el lugar de los hechos (en este caso el aula de clase) y con un diseño de investigación-acción, teniendo en cuenta que la aplicación de un modelo pedagógico en el proceso de enseñanza-aprendizaje puede presentar problemas y necesitan su resolución; y junto a existir estos inconvenientes en el proceso, presentar también opciones para el mejoramiento, con un enfoque descriptivo-correlacional, mediante el cual se pretende describir las características principales del desarrollo de las clases y relacionarlas con la parte teórica que sustentan el modelo y el presente estudio.

\subsection{Unidad de análisis}

La Unidad Educativa Salcedo consta de 66 docentes de planta, con un alumnado de 1323 estudiantes legalmente matriculados en el año lectivo 2019-2020, según datos de la secretaría de la institución. Se inició la investigación en los novenos años en las cuatro materias básicas del tronco común (Lengua y Literatura, Ciencias Naturales, Matemáticas, Estudios Sociales), para lo cual se tuvo el debido consentimiento tanto de docentes como de estudiantes y autoridades.

Se desarrolló la observación de clase en el noveno A, el cual consta de 31 estudiantes, y D que consta de 32 estudiantes, esto debido a la carga horaria de cada docente inmerso/a en esta investigación.

Para efectuar la encuesta al profesorado se tomó en cuenta las materias esenciales (tronco común); asimismo, las personas involucradas son quienes imparten clases a todos los paralelos desde octavo hasta décimo año, por lo que se optó en dichos educadores como referencia principal.

\subsection{Técnicas de recolección}

Para el análisis del modelo educativo constructivista en la Unidad Educativa Salcedo, se utilizó un instrumento de medición tomado y adaptado de Nieto (2007), este consta de veinte ítems y uno de opinión, distribuidos de forma aleatoria. El diseño operacional contiene dos factores, los cuales corresponden a los modelos pedagógicos: tradicional y constructivista, cada uno con un total de diez alternativas de respuesta. 
Este instrumento se aplicó a cuatro docentes que imparten las materias básicas en el noveno año de EGB de la Unidad Educativa Salcedo, permitiendo un total de ochenta respuestas.

Para la aplicación de este instrumento se utilizó la plataforma virtual de Google Drive en un formulario, para la contestación este tiene una escala de tipo Likert, ordinal y se tuvo en cuenta los siguientes parámetros de respuesta:

$\mathrm{A}=$ Acuerdo

$=$ Desacuerdo

$\mathrm{NA} / \mathrm{ND}=\mathrm{Ni}$ de acuerdo $/ \mathrm{Ni}$ en desacuerdo

$\mathrm{TA}=$ Total Acuerdo

$\mathrm{TD}=$ Total Desacuerdo

También se utilizó una ficha de observación de clase tomada y adaptada de la Unidad Educativa desde el año 2017, la cual fue aprobada por el Consejo Ejecutivo y la Junta Académica de esta, cuyo instrumento tiene una escala tipo Likert donde el evaluador marca con una $(\mathrm{x})$ dentro de la celda que corresponde a la valoración del indicador.

La observación de la clase se enfoca en cuatro aspectos del/la docente dentro de su hora clase: el primer aspecto es actividades previas; el segundo aspecto es proceso de enseñanza-aprendizaje, en el cual las dimensiones de observación son: dominio disciplinar y curricular, gestión del aprendizaje, desarrollo profesional y compromiso ético; el tercer aspecto es el desarrollo de los estándares del aprendizaje; y el cuarto y último aspecto es el clima del aula.

\subsection{Procesamiento de análisis}

Cada uno de los instrumentos utilizados brinda la información necesaria para la culminación de esta investigación y para el cumplimiento de los objetivos planteados. Una de las técnicas utilizadas fue la encuesta, la cual permitió conocer aspectos importantes sobre la enseñanza-aprendizaje de los involucrados en la investigación, este instrumento de medición fue utilizado con docentes de cada una de las cuatro áreas principales, el cual fue enviado vía correo electrónico a cada uno de ellos mediante un formulario de Google; en este debían leer la pregunta y escoger mediante su criterio, teniendo en cuenta los parámetros de respuesta $\mathrm{A}=$ Acuerdo, $\mathrm{D}=$ Desacuerdo, $\mathrm{NA} / \mathrm{ND}=\mathrm{Ni}$ de acuerdo $/ \mathrm{Ni}$ en desacuerdo, $\mathrm{TA}=$ Total Acuerdo, $\mathrm{TD}=$ Total Desacuerdo.

Junto a las veinte preguntas se adjuntó una pregunta extra, en la cual debían dar su opinión sobre la educación que se brinda en la Unidad Educativa Salcedo.

Una vez concluido el formulario se lo descargó en un archivo de Excel.

Es necesario tener en cuenta que este instrumento de medición tiene una distribución de preguntas de acuerdo con el modelo pedagógico constructivista y tradicional, se lo puede visualizar en el Apéndice A.

En lo que corresponde al segundo instrumento se usó la técnica de observación en cuanto a la utilización del modelo constructivista dentro del aula para tener un referente acerca de qué forma este se está aplicando y si se logra obtener algún cambio en el alumnado.

Esta ficha de observación se utilizó en cuatro clases (Matemáticas, Ciencias Naturales, Lengua y Literatura, Estudios Sociales) y a la vez fueron observadas por parte de las autoridades del plantel, con un representante de la Junta Académica. Es imperativo recalcar que esta observación se desarrolló sin previo aviso a cada docente, este instrumento está valorado con el cumplimiento e incumplimiento de ciertos parámetros metodológicos del/la docente en la hora de clase. La ficha de observación se le puede observar en los anexos en el Apéndice B. 


\section{Resultados y discusión}

Antes de iniciar con el análisis de los resultados hay que tener en cuenta una afirmación, la cual es muy cierta: que el deseo de ser docente está fuertemente marcado por la vocación (Sennett, 2009).

\subsection{Análisis de instrumento a docentes}

Con respecto a la averiguación elaborada al personal docente por medio de la encuesta virtual, la cual se puede visualizar en el enlace en el Apéndice $C$ que, de los 20 ítems formulados, 10 están direccionados al modelo educativo constructivista $(1,4,6,9,11,13,14,16,19,20)$ y los otros 10 direccionados al modelo educativo tradicional $(2,3,5,7,8,10,12,15,17,18)$.

Esto se formuló tras tener una conversación en la cual "la Doctora Jácome como Vicerrectora de la institución menciona, aunque usted no lo crea todavía se mantiene en algunos docentes el modelo tradicional" (N. Jácome, comunicación personal, 20 de noviembre del 2019).

Una vez tabulada la encuesta efectuada a cada docente, se puede apreciar claramente que no existe mayor diferencia en las respuestas y se concluye que de manera teórica cada docente tiene claras las principales características de un/a docente en el modelo pedagógico constructivista, como son crear iniciativa del estudiante, generar autonomía, utilizar materiales didácticos interactivos y manipulables, desafiar a la investigación, involucrarse con el estudiantado para generar su propio conocimiento con base en experiencias.

Así como lo menciona Zapata-Ros (2015) en la página 6 dentro del marco referencial por lo que se da a entender que el profesorado de la Unidad Educativa Salcedo conoce del modelo constructivista en la parte teórica, sabe cuáles pueden ser las estrategias adecuadas junto con métodos y técnicas para mejorar el estudio dentro del aula de clase, pero lastimosamente en algunos casos no lo lleva a la práctica, como menciona la Vicerrectora de la institución, sea por falta de iniciativa o temor a entrar en una forma de enseñanzaaprendizaje donde el estudiantado va a tener más autonomía, desarrollar su cognición, adquirir habilidades y oportunidades de aprender.

\subsection{Análisis de observación de clase}

Se ejecutaron observaciones áulicas mediante una ficha, la cual se divide en cuatro parámetros, los cuales son: actividades previas (A), proceso de enseñanza aprendizaje (B), desarrollo de los estándares de aprendizaje (C) y clima del aula de clase (D).

Para cada una de las asignaturas analizadas mediante la ficha de observación se obtuvieron los resultados que se exponen a continuación.

\subsubsection{Matemáticas}

La docente que dicta esta asignatura tiene 13 años de experiencia docente y cinco años de ellos lo ha trabajado en la Unidad Educativa Salcedo, de acuerdo con los parámetros evaluados se analiza que existe un $95 \%$ de aciertos, lo cual significa que esto es aceptable y está enfocada dentro del modelo constructivista, mas no es así en la parte B del proceso de enseñanza aprendizaje literal 5 (Utiliza técnicas activas para fortalecer el pensamiento crítico), literal 9 (Utiliza recursos didácticos coherentes con el objetivo propuesto), y en la parte $\mathrm{C}$ del desarrollo de los estándares de aprendizaje en el literal 3 (Exterioriza práctica de valores y de trabajo colaborativo), no cumplió con esos indicadores, pero a su vez la clase fue muy aceptable, por el manejo de esta y cumpliendo con el modelo constructivista en el cual se basa este análisis, la única recomendación que se 
ejecutó por parte de la autoridad fue que como en esta asignatura se utiliza números, se utilice diferente tipos de marcadores para mayor diferenciación del estudiantado en las operaciones que se realiza, por otra parte, hay que trabajar para mejorar los aspectos negativos mencionados, debido a que es parte fundamental del aprendizaje del estudiantado el trabajo colaborativo y a su vez siempre mencionar la importancia de mantener los valores dentro y fuera del aula.

\subsubsection{Ciencias Naturales}

El docente que dicta esta asignatura tiene siete años de experiencia y un año de docente en la Unidad Educativa Salcedo, en la observación de esta clase el docente cumplió con $40 \%$ de la ficha de observación, se encontraron varios inconvenientes en la hora clase, entre los cuales se pueden señalar: incumplimiento de la hora de inicio de clases, no organizó una ambientación del aula para favorecer el trabajo colaborativo, no hubo motivación, el estudiantado parecía cansado, nunca indagó acerca de los conocimientos previos del contenido por abordar, el docente generó siempre el conocimiento basado únicamente en el texto, no utilizó materiales didácticos, a lo que se une el hecho de que la letra no era muy legible en el pizarrón, no distribuyó acertadamente el tiempo de la clase para impartir los contenidos, no existió una participación activa del docente, aunque por otro lado mantuvo siempre la disciplina dentro del aula.

Por lo que se puede sintetizar que dicho docente utilizó un modelo tradicional en la clase, lo cual no permitió un buen ambiente y desarrollo de las actividades por parte del alumnado, lo que colocó así un límite a la interacción docente-estudiante.

\subsubsection{Estudios Sociales}

El docente que dicta esta asignatura tiene 10 años de experiencia y 3 en la Unidad Educativa Salcedo, en la observación de esta clase el docente cumplió con el 50 \% de la ficha de observación, encontrando algunos inconvenientes en la hora clase como que no hubo organización para favorecer el trabajo colaborativo, no hubo motivación ni al inicio ni en el transcurso de la clase, mantuvo medianamente el interés de sus estudiantes ya que hacía preguntas del tema tratado, la letra del docente no era comprensible teniendo en cuenta que el color del marcador no ayudaba a ello, sin embargo anotaba las partes principales, no utilizó material didáctico, utilizó el texto como parte fundamental de la clase y el docente generaba todo el conocimiento, no hubo una participación activa. En las partes positivas se puede mencionar que se mantuvo la disciplina, inició a la hora puntual y efectuaba una evaluación continua durante el transcurso de la clase.

Se puede concluir que todavía existe un predominio en la utilización del modelo tradicional por parte del docente al momento de impartir la clase y no hay la conexión suficiente entre sus estudiantes para llevar el proceso de enseñanza-aprendizaje de la mejor forma.

\subsubsection{Lengua y Literatura}

El docente que dicta esta asignatura tiene 26 años de experiencia y tres en la Unidad Educativa Salcedo, en la observación de esta clase el docente cumplió con el 50 \% de la ficha de observación, encontrando algunos inconvenientes en la hora clase como que no hubo organización para favorecer el trabajo colaborativo, no hubo motivación ni al inicio ni en el transcurso de la clase, no hubo interés del alumnado en el tema tratado, no hubo manejo del pizarrón, no hubo utilización de material didáctico, utilizó el texto como parte fundamental de la clase y el docente generaba todo el conocimiento, no hubo una participación activa del estudiantado en ningún momento, en las partes positivas se encontró que se mantuvo la disciplina, inició a la hora puntual, hubo la revisión de tareas al inicio. Al igual que los anteriores dos docentes, se puede concluir 
que todavía existe una utilización del modelo tradicional en la clase, algo que debe ser atendido para mejorar las actividades educativas.

A todas estas realidades dentro de la institución educativa principalmente en noveno año de EGB donde se desarrolló la investigación se debe tener en cuenta varios aspectos para poder implementar el modelo constructivista, así como mencionan Espejo y Sarmiento (2017) en la página 8 del marco referencial, cada docente de esta unidad educativa debe ser capaz de aplicar situaciones reales al estudiantado y al mismo tiempo estos sean capaces de brindar una solución rápida a la problemática planteada, logrando de esta manera desarrollar un mejor conocimiento cognitivo de cada uno.

\section{ConcLUSIONES}

El estudio permitió establecer las limitaciones fundamentales en la aplicación del modelo constructivista en el proceso enseñanza-aprendizaje en la unidad educativa salcedo.

Del estudio elaborada en la Unidad Educativa Salcedo en el noveno año de EGB se puede concluir que tres de cuatro docentes aún se encuentran inmersos en la enseñanza tradicional, lo cual impide que esta mejore dentro del ambiente áulico, esto detiene el desarrollo cognitivo del estudiantado y la calidad educativa de la institución.

El modelo pedagógico constructivista dentro del proceso de enseñanza aprendizaje es muy importante, por ende, el personal docente debe ser capaz de aplicarlo, porque interviene directamente en el desarrollo de habilidades y destrezas de cada estudiante.

$\mathrm{Al}$ alumnado, de manera general y no solo de noveno año, se le debe enseñar mediante métodos, técnicas o procesos apropiados donde este intervenga sean juegos, actividades colaborativas, exposiciones con materiales visuales, entre otros con la finalidad de tener toda la atención posible y obtengan la información compartida.

El estudiantado de noveno año tiene falencias en su aprendizaje por el descuido de este, la poca motivación docente, posiblemente en algunos casos la falta de compromiso de los padres de familia al no ser una guía desde su hogar y la utilización del modelo tradicionalista en la enseñanza, lo que hace las clases monótonas y aburridas para el estudiantado.

Dentro de la institución, y principalmente en noveno año, la enseñanza tradicionalista permanece en casi todo el profesorado, esto porque en gran parte son personas que sus bases se sustentan en aquello, se resisten al cambio por su misma edad al contrario de docentes jóvenes, no tienen capacitación adecuada, y existe el temor de aplicar un nuevo modelo para mejorar el aprendizaje.

\section{RECOMENDACIONES}

Las autoridades deben organizar capacitaciones permanentes hacia todo el profesorado en la aplicación del modelo constructivista, con la finalidad de proporcionar técnicas y métodos adecuados para mejorar la enseñanza aprendizaje del estudiantado.

Se debe elaborar un seguimiento a estudiantes y docentes que intervinieron en la encuesta para conocer si aceptaron las recomendaciones propuestas para mejorar sus técnicas y métodos dentro del aula de clase.

Las autoridades mediante el DECE deben conocer un poco más sobre las realidades del alumnado, así como que el profesorado debe motivar a sus estudiantes al inicio de sus clases para que esta sea más a mena y más llevadera.

El cuerpo docente debe autocapacitarse en el modelo constructivista y no solo esperar a las autoridades, existen distintos medios tecnológicos hoy en día para obtener información, cómo se puede aplicar en clase y qué alternativa se puede desarrollar para dejar lo tradicionalista. 


\section{Referencias Bibliográficas}

Campos, V. y Moya, R. (2011). La formación del profesional desde una concepción personalizada del proceso de aprendizaje. Cuadernos de Educación y desarrollo 3(28), 1-6. Recuperado de https://www.eumed.net/rev/ced/ 28/cpmr.pdf

Domínguez G. (2010) Las estrategias constructivistas en la enseñanza de la geografía. El ABP, Boca del Rio (Tesis de maestría). Universidad Veracruzana, México. Recuperado de https://bit.ly/312jZVA

Durán, O. (2015). Estrategias de enseñanza-aprendizaje que emplean los docentes de matemática en el Instituto Belga Guatemalteco. (Tesis maestría). Universidad Rafael Landívar, Guatemala. Recuperado de http://recursosbiblio .url.edu.gt/tesiseortiz/2015/05/83/Duran-Olga.pdf

Espejo, R. y Sarmiento, R. (2017). Manual de apoyo docente: Metodologías activas para el aprendizaje. Santiago, Chile: Universidad de Chile. Recuperado de https://www.slideshare.net/eraser/manual-de-apoyo-docente-metodolo gas-activas-para-el-aprendizaje

Kuiper R., Murdock N., Grant, N. (2010). Thinking strategies of baccalaureate nursing students prompted by selfregulated learning strategies. Journal of Nursing Education, 49(8), 429-436. https://doi.org/10.3928/0148483 4-20100430-01

Marrero, O. (2017). El proceso de enseñanza-aprendizaje por competencias. Una visión desde el enfoque sistémico. Congreso Universidad, 6(4), 28-46. Recuperado de http://revista.congresouniversidad.cu/index.php/rcu/articl e/view/837/786

Morales, H., y Irigoyen, A. (2016). El paradigma conductista y constructivista de la educación a través del Decálogo del Estudiante. Medicina Familiar, 18(2), 27-30. Recuperado de https://www.medigraphic.com/pdfs/medfam /amf-2016/amf162a.pdf

Nieto, L. (2007). Instrumento para identificar modelos Instrumento para identificar modelos pedagógicos en el Instituto Técnico pedagógicos en el Instituto Técnico Rafael Reyes de la ciudad de Duitama. Cuaderno de Lingüistica Hispana, (10), 189-205. Recuperado de https://www.redalyc.org/pdf/3222/322227484012.pdf

Pérez, A. y Morales, O. (2012). Metodología constructivista en el proceso de enseñanza - aprendizaje en el área de computación, (Tesis de licenciatura). Universidad Estatal de Milagro, Ecuador. Recuperado de https://bit.ly/3 axgnhv

Robalino, J. (2016). Modelo pedagógico constructivista y su influencia en el proceso de aprendizaje de los estudiantes de la Unidad Educativa Juan Benigno Vela, (Tesis de maestría). Universidad Técnica de Ambato, Ecuador. Recuperado de

Rosero, A. (2017). Constructivismo y prácticas escolares de las instituciones de Educación Intercultural Bilingüe de la Provincia de Cotopaxi. (Tesis de licenciatura). Universidad Politécnica Salesiana sede Quito, Ecuador. Recuperado de https://dspace.ups.edu.ec/bitstream/123456789/14753/1/UPS-QT12019.pdf

Saldarriaga, P., Bravo, G. y Loor, M., (2016). La teoría constructivista de Jean Piaget y su significación para la pedagogía contemporánea. Revista Cientifica Dominio de las Ciencias, 2, 127-137. Recuperado de https://dialnet.unirioja .es/descarga/articulo/5802932.pdf

Sennett, R. (2009). El artesano. Barcelona, España: Anagrama.

Serna, E. (2011). Los métodos y técnicas de enseñanza del constructivismo como medios para el desarrollo del pensamiento lógico, (Tesis de maestría). Universidad Andina Simón Bolívar, Ecuador. Recuperado de http://repositorio.uas b.edu.ec/handle/10644/3032

Serrano, J. y Pons, R. (2011). El constructivismo hoy: enfoques constructivistas en educación. Revista Electrónica de Investigación Educativa, 13(1),1-27. Recuperado de https://www.redalyc.org/pdf/155/15519374001.pdf

UNESCO. (2015). Enseñanza y aprendizaje, Lograr la calidad para todos, una mirada sobre América latina y el Caribe. Chile: Oficina Regional de Educación para América Latina y el Caribe (OREALC/UNESCO Santiago). Recuperado de https://bit.ly/327SxFo 
José Luis Amores Torres, et AL. Limitaciones del modelo constructivista en la enseñanZa-APrendizAJ...

Vera, E. (2009). El constructivismo aplicado en la enseñanza del área de ciencias sociales en el grupo 86 en la Institución Educativa Ciro Mendia. (Tesis de licenciatura). Universidad de Antioquia Facultad de Educación Medellín, Colombia. Recuperado de http://ayura.udea.edu.co:8080/jspui/bitstream/123456789/1570/1/PB0682.pdf

Zapata-Ros, M. (2015). Teorías y modelos sobre el aprendizaje en entornos conectados y ubicuos. Bases para un nuevo modelo teórico a partir de una visión crítica del "conectivismo.", Education In The Knowledge Society (EKS), 16(1), 69-102. doi: https://doi.org/10.14201/eks201516169102

\section{APÉNDICE A}

\section{INSTRUMENTO DE ENCUESTA REALIZADA A LOS DOCENTES}

\begin{tabular}{|c|c|c|c|c|c|}
\hline \multicolumn{6}{|c|}{ UNIDAD EDUCATIVA “SALCEDO" } \\
\hline \multicolumn{6}{|c|}{ Nombre: } \\
\hline \multicolumn{6}{|c|}{ Curso y Paralelo } \\
\hline \multicolumn{6}{|c|}{ Fecha: } \\
\hline \multicolumn{6}{|c|}{ Años de experiencia como Docente: } \\
\hline \multirow{2}{*}{$\mathrm{N}^{\circ}$} & \multirow{2}{*}{ CRITERIO PEDAGÓGICO } & \multicolumn{4}{|c|}{ VALORACIÓN } \\
\hline & & $\mathrm{D}$ & $\mathrm{NA} / \mathrm{ND}$ & $\mathrm{TA}$ & TD \\
\hline 1 & La construcción del conocimiento depende de las experiencias fuera del aula del estudiante & & & & \\
\hline 2 & Sin textos escolares no se puede enseñar. & & & & \\
\hline 3 & El docente es el que brinda todos sus conocimientos y es la autoridad dentro del aula. & & & & \\
\hline 4 & El aprendizaje se da a partir de los problemas de la vida diaria. & & & & \\
\hline 5 & Es necesario castigar al estudiante para que aprenda. & & & & \\
\hline 6 & La evaluación debe ser durante todo el proceso de enseñanza. & & & & \\
\hline 7 & El docente genera autoridad, disciplina, orden, características necesarias para generar aprendizaje. & & & & \\
\hline 8 & El dictado en parte esencial en el proceso de enseñanza - aprendizaje. & & & & \\
\hline 9 & Ningún estudiante está falto de conocimiento. & & & & \\
\hline 10 & Enseñar es involucrarse con el estudiante en su proceso de aprendizaje. & & & & \\
\hline 11 & La mejor manera de enseñar es la repetición, la memorización y la obediencia. & & & & \\
\hline 12 & 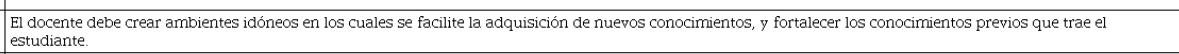 & & & & \\
\hline 13 & El diálogo, la concertación y el debate sobre distintos puntos de vista, asi como la lectura y la escritura son fundamentales para lograr un aprendizaje significativo. & & & & \\
\hline 14 & La mejor forma de generar aprendizaje en los estudiantes es clasificarlos de buenos y malos. & & & & \\
\hline 15 & El reto del buen docente es acompañar al estudiante en su proceso individual de aprendizaje. & & & & \\
\hline 16 & Usted como docente cree en el dicho: "La letra con sangre entra". & & & & \\
\hline 17 & El bajo rendimiento de los alumnos se genera por el quemeimportismo de ellos mismos. & & & & \\
\hline 18 & La generación del aprendizaje se lo puede dar de una manera colaborativa entre estudiantes. & & & & \\
\hline 19 & Se debe integrar, de manera flexible contenidos, objetivos e intereses, en función de cada estudiante. & & & & \\
\hline 20 & ¿El docente dentro del proceso enseñanza aprendizaje debe aplicar el modelo constructivista? & & & & \\
\hline 21 & De su opinión sobre la educación que se brinda en la Unidad Educativa salcedo & & & & \\
\hline
\end{tabular}




\section{APÉNDICE B}

FICHA DE OBSERVACIÓN DE CLASE AÑO LECTIVO 2019-2020

\begin{tabular}{|l|l|l|l|}
\hline \multicolumn{2}{|l|}{ DATOS INFORMATIVOS } \\
\hline Nombre de la Institución Educativa & \multicolumn{2}{l|}{$\begin{array}{l}\text { Unidad Educativa } \\
\text { "Salcedo" }\end{array}$} \\
\hline Nombre y Apellido /Docente & \multicolumn{2}{|l|}{} \\
\hline Asignatura & \multicolumn{2}{|l|}{} \\
\hline Años de Experiencia & & Paralelo & \\
\hline Curso & & Jornada & \\
\hline Número de estudiantes: & & Quimestre & \\
\hline Fecha: &
\end{tabular}

\section{OBJETIVO:}

Obtener información sobre la gestión del docente en el aula y de los aprendizajes de los estudiantes, para el mejoramiento continuo de la práctica pedagógica

Instrucciones:

El docente evaluador marca con una $(\mathrm{x})$ dentro de la celda que corresponde a la valoración del indicador, mediante la siguiente escala:

Cumple : SI

No Cumple : NO

\section{A. ACTIVIDADES PREVIAS}

\begin{tabular}{|l|c|c|}
\hline Indicadores & Sí & NO \\
\hline 1. Organiza la ambientación del aula para favorecer el trabajo colaborativo & & \\
\hline 2. Presenta el plan de clase al observador & & \\
\hline 3. Inicia su clase con puntualidad & & \\
\hline 4. Verifica el cumplimiento de las tareas (sellado o recepción) & & \\
\hline
\end{tabular}




\section{A. PROCESO DE ENSEÑANZA APRENDIZAJE DIMENSIONES: DOMINIO DISCIPLINAR Y CURRICULAR/ GESTIÓN DEL APRENDIZAJE/ DESARROLLO PROFESIONAL/ COMPROMISO ÉTICO}

\begin{tabular}{|l|l|l|}
\hline Indicadores & Sí & NO \\
\hline 1. Realiza ejercicios de motivación & & \\
\hline 2. Parte de los conocimientos previos & & \\
\hline 3. Da a conocer los objetivos de la clase & & \\
\hline 4. Mantiene el interés del estudiante en todo el proceso & \\
\hline 5. Utiliza Técnicas activas para fortalecer el pensamiento crítico & & \\
\hline 6. Domina el contenido de la clase & \\
\hline 7. Utiliza la pizarra de manera ordenada & \\
\hline 8. La letra es legible & & \\
\hline 9. Utiliza recursos didácticos coherentes con el objetivo propuesto & \\
\hline 10. $\quad$ Resume con los estudiantes los aspectos más relevantes de la clase & & \\
\hline 11. Utiliza el texto como parte fundamental de la clase & \\
\hline 12. & Distribuye el tiempo adecuadamente & \\
\hline 13. & & \\
\hline
\end{tabular}

\section{B. DESARROLLO DE LOS ESTANDARES DE APRENDIZAJE}

\begin{tabular}{|l|l|l|}
\hline Indicadores & Sí & NO \\
\hline 1. El estudiante demuestra en su desempeño el nivel de conocimiento & & \\
\hline 2. Participa activamente & & \\
\hline 3. Exterioriza práctica de valores y trabajo colaborativo & & \\
\hline 4. Expresa la comprensión de conceptos & & \\
\hline
\end{tabular}

\section{CLIMA DEL AULA}

\begin{tabular}{|l|l|l|}
\hline Indicadores & Sí & NO \\
\hline 1. Trata con respeto y amabilidad a los estudiantes & & \\
\hline 2. Promueve la participación & & \\
\hline 3. Mantiene la disciplina en el aula & & \\
\hline 4. Atiende las necesidades e inquietudes de los estudiantes & & \\
\hline
\end{tabular}

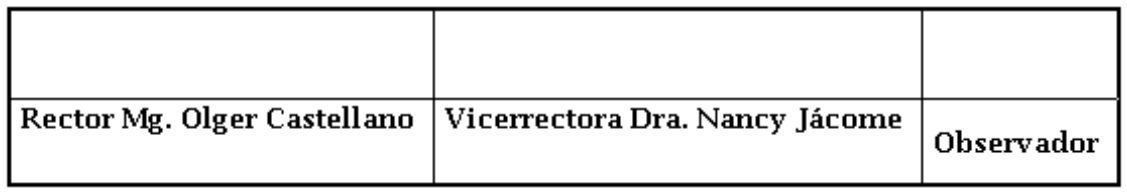




\section{APÉNDICE C}

RESULTADOS DE LA INVESTIGACIÓN

\section{ENCUESTA:}

DISPONIBLE EN:

https://forms.gle/crjrbbz2yGZYkTeh7

RESPUESTAS DE ENCUESTAS REALIZADAS A LOS DOCENTES

DISPONIBLE EN: https://drive.google.com/open?id=10nJ58zzIazswdcDyHKG8FsS3AvAaSG0P

OBSERVACIONES DE CLASE REALIZADAS A LOS DOCENTES

DISPONIBLES EN:

https://drive.google.com/open?id=1nYRI9KPXKdNCw8LL-9GtITskRboyukz_

CC BY-NC-ND 